\title{
Noether symmetries and the quantization of a Liénard-type nonlinear oscillator
}

\author{
G. Gubbiotti ${ }^{1}$ and M.C. Nucci ${ }^{1,2}$ \\ ${ }^{1}$ Dipartimento di Matematica e Informatica, Università di Perugia, 06123 Perugia, \\ Italy \\ ${ }^{2}$ INFN Sezione Perugia, Via Alessandro Pascoli, 06123 Perugia, Italy
}

\begin{abstract}
The classical quantization of a Liénard-type nonlinear oscillator is achieved by a quantization scheme (M. C. Nucci. Theor. Math. Phys., 168:997-1004, 2011) that preserves the Noether point symmetries of the underlying Lagrangian in order to construct the Schrödinger equation. This method straightforwardly yields the correct Schrödinger equation in the momentum space (V. Chithiika Ruby, M. Senthilvelan, and M. Lakshmanan. J. Phys. A: Math. Gen., 45:382002, 2012), and sheds light into the apparently remarkable connection with the linear harmonic oscillator.
\end{abstract}

\section{Introduction}

The classical method in the passage from classical to quantum mechanics is based on the substitution of the classical coordinates and momenta $\left(q_{i}, p_{i}\right)_{i=1, \ldots, N}$ with the quantum operators:

$$
q_{i} \rightarrow q_{i}, \quad p_{i} \rightarrow-\mathrm{i} \frac{\partial}{\partial q_{i}}
$$

However if the quantization of nonstandard Hamiltonians is pursued then ambiguity may raise due to ordering non-commuting factors. In such cases, the normal ordering method - as described in classical textbooks such [1,2] - and the Weyl quantization scheme [3] were devised.

Also since quantum mechanics is essentially a linear theory then problems arise when nonlinear canonical transformations are involved and there is no guarantee of consistency [4 7 . For a more recent perspective on the canonical transformations in quantum mechanics see [8] and references within.

In [9] it was inferred that Lie symmetries should be preserved if a consistent quantization is desired. In [10] [ex. 18, p. 433] an alternative Hamiltonian for the simple harmonic oscillator was presented. It is obtained by applying a nonlinear canonical transformation to the classical Hamiltonian of the harmonic oscillator. That alternative Hamiltonian was used in [11] to demonstrate what nonsense the usual quantization schemes produce. In [12] a quantization scheme that preserves the Noether symmetries was proposed and applied to Goldstein's example in order to derive the correct Schrödinger equation. In 13 the same quantization scheme was applied in order to quantize Calogero's goldfish system. 
Let us recall here the quantization scheme that preserves the Noether symmetries. We consider a linearizable system of second-order ordinary differential equations, i.e.:

$$
\ddot{\mathbf{x}}(t)=\mathbf{f}(t, \mathbf{x}, \dot{\mathbf{x}}), \quad \mathbf{x} \in \mathbb{R}^{N}
$$

which possess the maximal number of admissible Lie symmetries, namely $N^{2}+4 N+3$. In [14,15] it was proven that the maximal-dimension Lie symmetry algebra of a system of $N$ equations of second order is isomorphic to $\operatorname{sl}(N+2, \mathbb{R})$, and that the corresponding Noether symmetries generate a $\left(N^{2}+3 N+6\right) / 2$-dimensional Lie algebra $g^{V}$ whose structure (LeviMalceev decomposition and realization by means of a matrix algebra) was determined.

The new algorithm that yields the Schrödinger equation can be summarized as follows:

Step 1. Find the linearizing transformation which do not change the time, as prescribed in non-relativistic quantum mechanics:

$$
t \rightarrow t, \quad \mathbf{x} \rightarrow \mathbf{y}=\mathbf{y}(t, \mathbf{x})
$$

Step 2. Find a Lagrangian that admits the maximum number of Noether symmetries, namely $\left(N^{2}+3 N+6\right) / 2$.

Step 3. Apply the linearizing transformation $\mathbf{y}=\mathbf{y}(t, \mathbf{x})$ to the Schrödinger equation of the corresponding classical linear problem. This yields the Schrödinger equation corresponding to system (2).

Step 4. Check if the quantization is consistent with the classical properties of the system, namely find the Lie symmetries of the obtained Schrödinger equation:

$$
\Omega=T \partial_{t}+\sum_{i=1}^{n} X_{i} \partial_{x_{i}}+\Psi \partial_{\psi}
$$

and verify that $T \partial_{t}+\sum_{i=1}^{n} X_{i} \partial_{x_{i}}$ generate the Noether symmetries admitted by the Lagrangian of system (2).

Remark: Since the Schrödinger equation is homogeneous and linear, it admits also the the homogeneity symmetry $\psi \partial_{\psi}$, and the linearity symmetry $\Omega_{\Psi}=\Psi \partial_{\psi}$, where $\Psi$ is any particular solution of the Schrödinger equation itself.

In this paper we apply this new quantization algorithm to a linearizable Liénard equation, i.e.

$$
\ddot{x}+k x \dot{x}+\frac{k^{2}}{9} x^{3}+\omega^{2} x=0,
$$

that has been recently quantized in momentum space [16].

In Section 2 we recall the properties of the linearizable Liénard equation (15). In Section 3 we consider the classical analogue of the momentum representation of equation (5) as given in [16] and after showing that is linearizable we quantize it by preserving the Noether symmetries. In Section 4 a comparison between the Noether quantization method and that applied in [16] is given. The last Section contains some final remarks. 


\section{Classical properties of the Liénard equation (5)}

The one-dimensional nonlinear oscillator (5) is a special case of the general Liénard equation, i.e.

$$
\ddot{x}+f(x) \dot{x}+g(x)=0,
$$

introduced more than 75 years ago [17,18] for modeling electrical circuits. Since then Liénard equations have been applied to many different areas even in biology [19].

The particular Liénard equation (5) has been extensively studied by many authors, the most recent papers being [20 23]. In [24 26] Lie group analysis was applied to (6) and it was shown that equation (5) is the only linearizable equation among those in (6). In fact equation (5) was found to admit an eight-dimensional Lie point symmetry algebra generated by the following operators:

$$
\begin{aligned}
\Gamma_{1} & =x \partial_{t}-\left(\frac{1}{3} x^{3} k+\frac{3 \omega^{2}}{k} x\right) \partial_{x} \\
\Gamma_{2} & =\sin (\omega t) x \partial_{t}-\left(\frac{k}{3} \sin (\omega t) x^{3}-\omega \cos (\omega t) x^{2}\right) \partial_{x} \\
\Gamma_{3} & =\cos (\omega t) x \partial_{t}-\left(\frac{k}{3} \cos (\omega t) x^{3}+\omega \sin (\omega t) x^{2}\right) \partial_{x} \\
\Gamma_{4} & =\left(\frac{3 \omega}{k} \cos (2 \omega t)-\sin (2 \omega t) x\right) \partial_{t} \\
& +\left[\left(\frac{k}{3} x^{3}-\frac{3 \omega^{2}}{k} x\right) \sin (2 \omega t)-2 \omega x^{2} \cos (2 \omega t)\right] \partial_{x} \\
\Gamma_{5} & =-\left(\frac{3 \omega}{k} \sin (2 \omega t)+\cos (2 \omega t) x\right) \partial_{t} \\
& -\left[\left(\frac{k}{3} x^{3}-\frac{3 \omega^{2}}{k} x\right) \cos (2 \omega t)+2 \omega x^{2} \cos (2 \omega t)\right] \partial_{x} \\
\Gamma_{6} & =\cos (\omega t) \partial_{t}+\left(\omega \sin (\omega t) x-\frac{3 \omega^{2}}{k} \cos (\omega t)\right) \partial_{x}, \\
\Gamma_{7} & =-\sin (\omega t) \partial_{t}+\left(\omega \cos (\omega t) x+\frac{3 \omega^{2}}{k} \sin (\omega t)\right) \partial_{x} \\
\Gamma_{8} & =\partial_{t} .
\end{aligned}
$$

Following Lie [27, the linearizing transformation is given by finding a two-dimensional abelian intransitive subalgebra and put it into the canonical form $\partial_{\tilde{x}}, \tilde{t} \partial_{\tilde{x}}$. Since a twodimensional abelian intransitive subalgebra is that generated by

$$
k \Gamma_{2}-3 \omega \Gamma_{6}, \quad k \Gamma_{3}-3 \omega \Gamma_{7}
$$

then the point transformation that takes (5) into the one-dimensional free-particle

$$
\frac{\mathrm{d}^{2} \tilde{x}}{\mathrm{~d} \tilde{t}^{2}}=0
$$


is

$$
\tilde{t}=\frac{k x \cos (\omega t)+3 \omega \sin (\omega t)}{k x \sin (\omega t)-3 \omega \cos (\omega t)}, \quad \tilde{x}=-\frac{1}{9 \omega^{2}} \frac{x}{k x \sin (\omega t)-3 \omega \cos (\omega t)} .
$$

Indeed the general solution of (5) is known to be

$$
x=\frac{9 \omega^{3} A \sin (\omega t+\delta)}{k-3 \omega^{2} k A \cos (\omega t+\delta)},
$$

with $A$ and $\delta$ arbitrary constants.

Thus equation (51) represents a non-linear oscillator - at least if $|A|<1 /\left(3 \omega^{2}\right)$ - and should be related to the linear harmonic oscillator. In [20] it was shown that the nonlocal transformation

$$
U=x e^{\frac{k}{3} \int x(\tau) \mathrm{d} \tau}
$$

takes equation (5) into the linear harmonic oscillator:

$$
\ddot{U}+\omega^{2} U=0 .
$$

Moreover the substitution of the general solution (11) into (12) yielded the following canonical transformation between (5) and (13):

$$
x=\frac{U}{1-\frac{k}{3 \omega^{2}} P}, \quad p=P\left(1-\frac{k}{6 \omega^{2}} P\right) .
$$

Here we show that (12) yields the canonical transformation (14) without knowing the general solution of (5).

In [16] the following Hamiltonian for equation (5) was presented:

$$
H=\frac{9 \omega^{4}}{2 k^{2}}\left[2-\frac{2 k}{3 \omega^{2}} p-2\left(1-\frac{2 k}{3 \omega^{2}} p\right)^{\frac{1}{2}}+\frac{k^{2} x^{2}}{9 \omega^{2}}\left(1-\frac{2 k}{3 \omega^{2}} p\right)\right] .
$$

with the restriction $-\infty<p \leq 3 \omega^{2} / 2 k$, and also the corresponding Lagrangian was derived, i.e.:

$$
L=\frac{27 \omega^{6}}{2 k^{2}} \frac{1}{k \dot{x}+\frac{k^{2}}{3} x^{2}+3 \omega^{2}}+\frac{3 \omega^{2}}{2 k} \dot{x}-\frac{9 \omega^{2}}{2 k^{2}} .
$$

We observe that the last two terms of the Lagrangian (16) represent the total derivative of the function:

$$
G=\frac{3 \omega^{2}}{2 k} x-\frac{9 \omega^{4}}{2 k^{2}} t
$$

This choice implies that the momentum is given by the equation:

$$
p \equiv \frac{\partial L}{\partial \dot{x}}=-\frac{27 \omega^{6}}{2 k} \frac{1}{\left(k \dot{x}+\frac{k^{2}}{3} x^{2}+3 \omega^{2}\right)^{2}}+\frac{3 \omega^{2}}{2 k},
$$


and consequently the velocity $\dot{x}$ is given by:

$$
\dot{x}=-\frac{k}{3} x^{2}+3 \omega^{2} \frac{1-\sqrt{1-\frac{2 k}{3 \omega^{2}} p}}{k \sqrt{1-\frac{2 k}{3 \omega^{2}} p}} .
$$

Remark: Although the addition of the total derivative of the particular function $G$ in (17) may seem useless, it actually allows to replace the otherwise ambiguous term $\sqrt{-p}$ with $\sqrt{1-\frac{2 k}{3 \omega^{2}} p}$.

Apart an unessential multiplicative constant and the addition of the total derivative of $G$ in (17), the Lagrangian (16) was determined in [28] by means of the Jacobi Last Multiplier [29 32 as a particular case of the Lagrangian for the general Liénard equation (6), i.e.:

$$
L=\left(\dot{x}+\frac{g(x)}{\alpha f(x)}\right)^{2-1 / \alpha}+\frac{\mathrm{d}}{\mathrm{d} t} G(t, x) .
$$

when the following relationship holds between $f(x)$ and $g(x)$ :

$$
\frac{\mathrm{d}}{\mathrm{d} x}\left(\frac{g(x)}{f(x)}\right)=\alpha(1-\alpha) f(x)
$$

with $\alpha$ a constant $\neq 1$.

In the case of equation (5) it was shown in 28, that the relationship (21) holds, and consequently the following function was determined:

$$
q=\frac{x}{\dot{x}+\frac{k}{3} x^{2}+\frac{3 \omega^{2}}{k}}
$$

such that $q=x e^{\frac{k}{3} \int x(\tau) \mathrm{d} \tau}$ and satisfies the linear harmonic oscillator equation:

$$
\ddot{q}+\omega^{2} q=0 .
$$

Therefore the nonlocal transformation (12) is a contact transformation and indeed canonical. Substituting $p$ as given in (18) into (22) yields:

$$
x=-\frac{3 \omega^{2} q}{k \sqrt{1-\frac{2 k}{3 \omega^{2}} p} .}
$$

Let us search for the new momentum $s$ by using a generating function of type $f_{3}=f_{3}(q, p)$ [10]. Then the following equation:

$$
\frac{\partial f_{3}}{\partial p} \equiv x=-\frac{3 \omega^{2} q}{k \sqrt{1-\frac{2 k}{3 \omega^{2}} p}}
$$


yields the generating function:

$$
f_{3}=Q(q)+9 \omega^{4} q \sqrt{1-\frac{2 k}{3 \omega^{2}} p}
$$

with $Q(q)$ an arbitrary function of $q$. Then substituting $f_{3}$ into

$$
\frac{\partial f_{3}}{\partial q}=s,
$$

yields:

$$
p=-\frac{k^{3}}{54 \omega^{6}} s^{2}+\frac{3 \omega^{2}}{2 k}
$$

where we have assumed $Q=0$ for the sake of simplicity. These new canonical variables $q, s$ transform the Hamiltonian (15) into the new Hamiltonian:

$$
K=\frac{1}{2}\left(\frac{k s}{9 \omega^{2}}+\frac{\omega^{2}}{k}\right)^{2}+\frac{9 \omega^{2}}{2 k^{2}} q^{2}
$$

which is connected to that of the linear harmonic oscillator by means of the following linear canonical transformation:

$$
\tilde{s}=\frac{3 \omega^{2}}{k} s-\frac{9 \omega^{2}}{k^{2}}, \quad \tilde{q}=\frac{k}{3 \omega^{2}} q,
$$

and thus the canonical transformation that takes (5) into the linear harmonic oscillator is recovered.

\section{Quantization of (5) in momentum space}

In [16] the quantization problem of (5) was tackled in the momentum representation since the Hamiltonian (15) is quadratic in $x$. The von Roos' quantization scheme [33, 34] was then applied.

Instead we begin with the classical equation and then apply the Noether symmetry quantization.

The canonical transformation

$$
(x, p) \rightarrow(X, P)=(p,-x)
$$

transforms the Hamiltonian (15) into the "inverted" Hamiltonian

$$
\tilde{H}=\frac{9 \omega^{4}}{2 k^{2}}\left[2-\frac{2 k}{3 \omega^{2}} X-2\left(1-\frac{2 k}{3 \omega^{2}} X\right)^{\frac{1}{2}}+\frac{k^{2} P^{2}}{9 \omega^{2}}\left(1-\frac{2 k}{3 \omega^{2}} X\right)\right]
$$

The corresponding Lagrangian is:

$$
\tilde{L}=\frac{\dot{X}^{2}}{2 \omega^{2}\left(1-\frac{2 k}{3 \omega^{2}} X\right)}-\frac{9 \omega^{4}}{2 k^{2}}\left(1-\sqrt{1-\frac{2 k}{3 \omega^{2}} X}\right)^{2} .
$$


and its Lagrangian equation is:

$$
\ddot{X}=\frac{3 \omega^{4}}{k}\left(1-\frac{2 k}{3 \omega^{2}} X-\sqrt{1-\frac{2 k}{3 \omega^{2}} X}\right)-\frac{k \dot{X}^{2}}{3 \omega^{2}\left(1-\frac{2 k}{3 \omega^{2}} X\right)} .
$$

Using the REDUCE programs [35] we determine that the Lie symmetry algebra admitted by equation (34) is generated by the following operators:

$$
\begin{aligned}
\Xi_{1}= & \partial_{t}, \\
\Xi_{2}= & \cos (2 \omega t) \partial_{t}+\sin (2 \omega t) \frac{3 \omega^{3}}{k}\left[1-\frac{2 k}{3 \omega^{2}} X-\sqrt{1-\frac{2 k}{3 \omega^{2}} X}\right] \partial_{X} \\
\Xi_{3}= & \sin (2 \omega t) \partial_{t}-\cos (2 \omega t) \frac{3 \omega^{3}}{k}\left[1-\frac{2 k}{3 \omega^{2}} X-\sqrt{\left.1-\frac{2 k}{3 \omega^{2}} X\right]} \partial_{X}\right. \\
\Xi_{4}= & \sqrt{1-\frac{2 k}{3 \omega^{2}} X \cos (\omega t) \partial_{X}} \\
\Xi_{5}= & \sqrt{1-\frac{2 k}{3 \omega^{2}} X \sin (\omega t) \partial_{X}} \\
\Xi_{6}= & \cos (\omega t)\left(\sqrt{\left.1-\frac{2 k}{3 \omega^{2}} X-1\right) \partial_{t}}\right. \\
& +\frac{3 \omega^{3}}{k} \sin (\omega t)\left(\sqrt{1-\frac{2 k}{3 \omega^{2}} X}-2\right)\left(1-\frac{2 k}{3 \omega^{2}} X\right) \partial_{X}, \\
\Xi_{7}= & \sin (\omega t)\left(\sqrt{\left.1-\frac{2 k}{3 \omega^{2}} X-1\right) \partial_{t}}\right. \\
& -\frac{3 \omega^{3}}{k} \cos (\omega t)\left(\sqrt{1-\frac{2 k}{3 \omega^{2}} X}-2\right)\left(1-\frac{2 k}{3 \omega^{2}} X\right) \partial_{X}, \\
\Xi_{8}= & \sqrt{1-\frac{2 k}{3 \omega^{2}} X\left(1-\sqrt{1-\frac{2 k}{3 \omega^{2}} X}\right) \partial_{X} .}
\end{aligned}
$$

Obviously equation (34) is linearizable and the operators $\Xi_{i}$ give a representation of the Lie algebra $s l(3, \mathbb{R})[27$. Therefore the Noether symmetry quantization can be applied step by step.

Step 1. Let us find the linearizing transformation. A two-dimensional abelian intransitive subalgebra is provided by $\Xi_{4}$ and $\Xi_{5}$ and thus the linearizing transformation that takes (34) into the free particle equation:

$$
\frac{\mathrm{d}^{2} \xi(\tau)}{\mathrm{d} \tau^{2}}=0
$$

is given by

$$
\tau=\tan (\omega t), \quad \xi=\omega \sqrt{\frac{6}{k}} \frac{1-\sqrt{1-\frac{2 k}{3 \omega^{2}} X}}{\cos (\omega t)} .
$$


Unfortunately this transformation involves changing the time $t$.

However we recall that the point transformation between the free particle (36) and the linear harmonic oscillator

$$
\frac{\mathrm{d}^{2} Z(t)}{\mathrm{d} t^{2}}+\omega^{2} Z(t)=0
$$

is given by:

$$
\tau=\tan (\omega t), \quad \xi=\frac{Z}{\cos (\omega t)} .
$$

Then it is easy to show that the transformation 1 :

$$
\eta=\omega \sqrt{\frac{6}{k}}\left(1-\sqrt{1-\frac{2 k}{3 \omega^{2}} X}\right)
$$

takes equation (34) into the linear harmonic oscillator:

$$
\ddot{\eta}+\omega^{2} \eta=0 .
$$

Thus the linearization transformation (40) yields the general solution of equation (34), i.e.:

$$
X(t)=\frac{3 \omega^{2}}{2 k}\left[1-\left(1-\frac{1}{\omega} \sqrt{\frac{k}{6}} A \sin (\omega t+\delta)\right)^{2}\right],
$$

where $A$ and $\delta$ are two arbitrary constants.

Remark: Equation (34) and Liénard equation (5) are examples of nonlinear oscillators whose amplitude do not depend on the frequency, unlike other famous nonlinear oscillators, e.g. the Mathews-Lakshmanan oscillator [36, 37].

Step 2. The Lagrangian (33) admits five Noether point symmetries, namely $\Xi_{i}$ with $i=$ $1, \ldots, 5$ in (35).

Step 3. Let us consider the Schrödinger equation for the linear harmonic oscillator:

$$
2 \mathrm{i} \widetilde{\Phi}_{t}+\widetilde{\Phi}_{\eta \eta}-\omega^{2} \eta^{2} \widetilde{\Phi}=0
$$

with $\widetilde{\Phi}=\widetilde{\Phi}(t, \eta)$. Then applying the transformation (40) we obtain the following Schrödinger equation :

$$
2 \mathrm{i} \widetilde{\Phi}_{t}+\frac{3 \omega^{2}}{2 k}\left(1-\frac{2 k}{3 \omega^{2}} X\right) \widetilde{\Phi}_{X X}-\frac{1}{2} \widetilde{\Phi}_{X}-\frac{6 \omega^{4}}{k}\left(1-\sqrt{1-\frac{2 k}{3 \omega^{2}} X}\right)^{2} \widetilde{\Phi}
$$

with $\widetilde{\Phi}=\widetilde{\Phi}(t, X)$. The following transformation eliminates the first derivative of $\Phi$ with respect to $X$ in (44):

$$
\Phi(t, X)=\frac{\widetilde{\Phi}(t, X)}{\left[3 \omega^{2}\left(1-\frac{2 k}{3 \omega^{2}} X\right)\right]^{\frac{1}{4}}}
$$

\footnotetext{
${ }^{1}$ We insert a constant factor $\omega / \sqrt{k}$ in order to give $\eta$ the same dimension as $\sqrt{x}$.
} 
and hence the final form of the Schrödinger equation is:

$$
\begin{aligned}
2 \mathrm{i} \Phi_{t} & +9\left(1-\frac{2 k}{3 \omega^{2}} X\right) \Phi_{X X} \\
& +\left[\frac{3 k^{2}}{4 \omega^{4}\left(1-\frac{2 k}{3 \omega^{2}} X\right)}-\frac{2 \omega^{6}}{k^{2}}\left(1-\frac{k}{3 \omega^{2}} X\right) \sqrt{1-\frac{2 k}{3 \omega^{2}} X}\right] \Phi
\end{aligned}
$$

Step 4. We now check the classical consistency of the Schrödinger equation (46). Using the REDUCE programs [35] we find that its Lie point symmetries are generated by the following operators:

$$
\begin{aligned}
& \Omega_{1}=\Xi_{1}, \\
& \Omega_{2}=\Xi_{2}+\left[\frac{\omega}{2} \sin (2 \omega t)-\mathrm{i} \omega^{2} \cos (2 \omega t)\left(1-\sqrt{1-\frac{2 k}{3 \omega^{2}} X}\right)^{2}\right] \frac{\Phi}{\sqrt{1-\frac{2 k}{3 \omega^{2}} X}} \partial_{\Phi}, \\
& \Omega_{3}=\Xi_{3}-\left[\frac{\omega}{2} \cos (2 \omega t)+\mathrm{i} \omega^{2} \sin (2 \omega t)\left(1-\sqrt{1-\frac{2 k}{3 \omega^{2}} X}\right)^{2}\right] \frac{\Phi}{\sqrt{1-\frac{2 k}{3 \omega^{2}} X}} \partial_{\Phi}, \\
& \left.\Omega_{4}=\Xi_{4}-\left[\frac{k \cos (\omega t)}{6 \omega^{2} \sqrt{1-\frac{2 k}{3 \omega^{2}} X}}+2 \mathrm{i} \omega\left(1-\sqrt{1-\frac{2 k}{3 \omega^{2}} X}\right) \sin (\omega t)\right] \Phi \partial_{\Phi}\right] \\
& \Omega_{5}=\Xi_{5}-\left[\frac{k \sin (\omega t)}{6 \omega^{2} \sqrt{1-\frac{2 k}{3 \omega^{2}} X}}-2 \mathrm{i} \omega\left(1-\sqrt{1-\frac{2 k}{3 \omega^{2}} X}\right) \cos (\omega t)\right] \Phi \partial_{\Phi} \\
& \Omega_{6}=\Phi \partial_{\Phi}, \\
& \Omega_{\chi}=\chi \partial_{\Phi},
\end{aligned}
$$

where $\chi$ is any solution of (46).

It was shown in [16] that the spectrum of the Liénard equation in momentum space consists of two parts, one positive and one negative.

The positive part is:

$$
E_{n}=\omega\left(n+\frac{1}{2}\right), \quad n \in \mathbb{N},
$$

which is the spectrum of the quantum harmonic oscillator. The eigenfunctions of this part of the spectrum satisfy the boundary conditions:

$$
\begin{array}{r}
\lim _{X \rightarrow-\infty} \Phi(t, X)=0, \quad \text { for every } t \in \mathbb{R}_{+}, \\
\Phi\left(t, \frac{3 \omega^{2}}{2 k}\right)=0, \quad \text { for every } t \in \mathbb{R}_{+} .
\end{array}
$$


The negative part is:

$$
E_{n^{-}}=-\omega\left(n+\frac{1}{2}\right), \quad n \in \mathbb{N},
$$

which is again the spectrum of the quantum harmonic oscillator, but with a negative sign. The eigenfunctions of this part of the spectrum satisfy the boundary conditions:

$$
\begin{aligned}
\lim _{X \rightarrow+\infty} \Phi(t, X) & =0, \quad \text { for every } t \in \mathbb{R}_{+}, \\
\Phi\left(t, \frac{3 \omega^{2}}{2 k}\right) & =0, \quad \text { for every } t \in \mathbb{R}_{+} .
\end{aligned}
$$

In [38 41] and more recently in [11] it was shown how to find the eigenfunctions and the eigenvalues of the Schrödinger equation by means of its admitted Lie symmetries.

We apply this method to the Schrödinger equation (46) and find the same results as in [16].

Let us rewrite the Lie point symmetries (47) of equation (46) in complex form, i.e.:

$$
\begin{aligned}
& \Sigma_{1}=\mathrm{i} \partial_{t}, \\
& \Sigma_{2 \pm}=e^{ \pm 2 \mathrm{i} \omega t} \partial_{t} \mp \mathrm{i} e^{ \pm 2 \mathrm{i} \omega t} \frac{3 \omega^{3}}{k}\left(1-\sqrt{1-\frac{2 k}{3 \omega^{2}} X}\right) \sqrt{1-\frac{2 k}{3 \omega^{2}} X} \partial_{X} \\
& +\frac{\mathrm{i}}{2} e^{ \pm 2 \mathrm{i} \omega t}\left[\frac{24 \omega^{3}-24 \omega^{3} \sqrt{1-\frac{2 k}{3 \omega^{2}} X}-k+8 k X \omega \sqrt{1-\frac{2 k}{3 \omega^{2}} X}-16 k \omega X}{\omega k \sqrt{1-\frac{2 k}{3 \omega^{2}} X}}\right] \Phi \partial_{\Phi} \\
& \Sigma_{3 \pm}=e^{ \pm \mathrm{i} \omega} \sqrt{1-\frac{2 k}{3 \omega^{2}} X} \partial_{X} \\
& \mp e^{ \pm \mathrm{i} \omega^{3}} 2 \omega\left[\left(1-\sqrt{1-\frac{2 k}{3 \omega^{2}} X}\right)+\frac{k}{12 \sqrt{3} \omega^{4}} \frac{1}{\sqrt{1-\frac{2 k}{3 \omega^{2}} X}}\right] \Phi \partial_{\Phi} \\
& \Sigma_{4}=\Phi \partial_{\Phi} \\
& \Sigma_{\chi}=\chi \partial_{\Phi}
\end{aligned}
$$

The operators $\Sigma_{3 \pm}$ act as creation and annihilation operators, namely in the case of the boundary conditions (48) $\Sigma_{3+}$ is the annihilation operator and $\Sigma_{3-}$ is the creation operator, and viceversa in the case of the boundary conditions (49).

Let us now consider the case of the boundary conditions (48). The invariant surface of the operator $\Sigma_{3+}$ is given by

$$
F(t, X, \Phi)=f\left(t, \Phi \frac{e^{-\frac{2 \omega}{k}\left(3 \omega^{2} \sqrt{1-\frac{2 k}{3 \omega^{2}} X}+k X\right)}}{\left(1-\frac{2 k}{3 \omega^{2}} X\right)^{\frac{1}{4}}}\right)=0,
$$

which if solved with respect to $\Phi$ yields

$$
\Phi(t, X)=T(t)\left(1-\frac{2 k}{3 \omega^{2}} X\right)^{\frac{1}{4}} e^{\frac{2 \omega}{k}\left(3 \omega^{2} \sqrt{1-\frac{2 k}{3 \omega^{2}} X}+k X\right)}
$$


with $T(t)$ arbitrary function of $t$. Substituting this solution into the Schrödinger equation (46) yields $T(t)=e^{-\frac{1}{2} \mathrm{it}}$ and thus the ground state solution is:

$$
\Phi_{0}(t, X)=\left(1-\frac{2 k}{3 \omega^{2}} X\right)^{\frac{1}{4}} e^{-\frac{1}{2} \mathrm{i} t+\frac{2 \omega}{k}\left(3 \omega^{2} \sqrt{1-\frac{2 k}{3 \omega^{2}} X}+k X\right)} .
$$

This solution $\Phi_{0}(t, X)$ satisfies the boundary conditions (48) and is indeed the ground state 2 since

$$
\left[\Sigma_{3+}, \Sigma_{\Phi_{0}}\right]=0 .
$$

Thus there are no states under $\Phi_{0}(t, X)$.

The operator $\Sigma_{1}$ acts like an eigenvalue operator since:

$$
\Sigma_{1} \Phi_{0}=\frac{\omega}{2} \Phi_{0}
$$

which yields the ground energy $E_{0}=\omega / 2$, just like the usual quantum harmonic oscillator.

We use the creation operator $\Sigma_{3-}$ and $\Sigma_{\Phi_{0}}$ in order to construct the higher states. Since the commutator:

$$
\left[\Sigma_{3-}, \Sigma_{\Phi_{0}}\right]=4 \omega^{2} e^{-\frac{3}{2} \mathrm{i} \omega t+\frac{2 \omega}{k}\left(3 \omega^{2} \sqrt{1-\frac{2 k}{3 \omega^{2}} X}+k X\right)}\left(1-\frac{2 k}{3 \omega^{2}} X\right)^{\frac{1}{4}}\left(\sqrt{1-\frac{2 k}{3 \omega^{2}} X}-1\right) \partial_{\Phi},
$$

then:

$$
\Phi_{1}(t, X)=4 \omega^{2} e^{-\frac{3}{2} i \omega t+\frac{2 \omega}{k}\left(3 \omega^{2} \sqrt{1-\frac{2 k}{3 \omega^{2}} X}+k X\right)}\left(1-\frac{2 k}{3 \omega^{2}} X\right)^{\frac{1}{4}}\left(\sqrt{1-\frac{2 k}{3 \omega^{2}} X}-1\right)
$$

is another solution of (46) that satisfies the boundary conditions (48) and has a greater energy eigenvalue $E_{1}=\frac{3 \omega}{2}$ given by:

$$
\Sigma_{1} \Phi_{1}=\frac{3 \omega}{2} \Phi_{1}
$$

If we evaluate the commutator between $\Sigma_{3+}$ and $\Sigma_{\Phi_{1}}$ then we obtain a multiple of $\Phi_{0}$, i.e.:

$$
\left[\Sigma_{3+}, \Sigma_{\Phi_{1}}\right]=-4 k \omega \Phi_{0} \partial_{\Phi}
$$

and thus we have constructed the first excited state.

Iterating the process yields all the eigenfunctions, i.e.:

$$
\left[\Sigma_{3-}, \Sigma_{\Phi_{n-1}}\right]=\Phi_{n} \partial_{\Phi}=\Sigma_{\Phi_{n}} .
$$

Since we have proven that $\Sigma_{3+}$ acts as the annihilation operator for the first excited state, then we can easily show by means of Jacobi identity 3 that this holds true for every $n \in \mathbb{N}$, i.e.:

$$
\begin{aligned}
{\left[\Sigma_{3+}, \Sigma_{\Phi_{n}}\right] } & =\left[\Sigma_{3+},\left[\Sigma_{3-}, \Sigma_{\Phi_{n-1}}\right]\right] \\
& =\left[\left[\Sigma_{\Phi_{n-1}}, \Sigma_{3+}\right], \Sigma_{3-}\right]+\left[\left[\Sigma_{3+}, \Sigma_{3-}\right], \Sigma_{\Phi_{n-1}}\right] \\
& =-\kappa\left[\Sigma_{\Phi_{n-2}}, \Sigma_{3-}\right]=\kappa \Sigma_{\Phi_{n-1}}
\end{aligned}
$$

\footnotetext{
${ }^{2}$ Apart from an unessential normalization constant.

${ }^{3}$ And by means of

$$
\left[\Sigma_{3+}, \Sigma_{3-}\right]=4 k \omega \Sigma_{4}
$$
}


where $\kappa$ is a constant.

The generic eigenvalue and eigenfunction can be derived in the following manner. We evaluate the commutator between $\Sigma_{3-}$ and $\Sigma_{\chi}$, where $\chi$ is a generic solution of (46):

$$
\begin{aligned}
{\left[\Sigma_{3-}, \Sigma_{\chi}\right]=\frac{e^{-\mathrm{i} \omega t}}{2 \omega \sqrt{3} \sqrt{1-\frac{2 k}{3 \omega^{2}} X}} } & {\left[\left(12 \omega^{3}+k-8 k \omega X-12 \omega^{3} \sqrt{1-\frac{2 k}{3 \omega^{2}} X}\right) \chi\right.} \\
& \left.+\left(6 \omega^{2}-4 k X\right) \chi_{X}\right] \partial_{\Phi} .
\end{aligned}
$$

We define the operator $\hat{O}$ :

$$
\hat{O}=\frac{1}{2 \omega \sqrt{3} \sqrt{1-\frac{2 k}{3 \omega^{2}} X}}\left[12 \omega^{3}+k-8 k \omega X-12 \omega^{3} \sqrt{1-\frac{2 k}{3 \omega^{2}} X}+\left(6 \omega^{2}-4 k X\right) \partial_{X}\right]
$$

and then beginning with the ground state $\Phi_{0}$ generate the $n$th eigenfunction by using the iteration procedure (60), i.e.:

$$
\begin{aligned}
\Phi_{1} & =e^{-\mathrm{i} \omega t} \hat{O} \Phi_{0}, \\
\Phi_{2} & =e^{-\mathrm{i} \omega t} \hat{O} \Phi_{1}=e^{-2 \mathrm{i} \omega t} \hat{O}^{2} \Phi_{0}, \\
& \vdots \\
\Phi_{n} & =e^{-\mathrm{i} \omega t} \hat{O} \Phi_{n-1}=e^{-\mathrm{i} n \omega t} \hat{O}^{n} \Phi_{0} .
\end{aligned}
$$

Since the operator $\hat{O}$ acts on $X$ only, then

$$
\Phi_{n}=e^{-\mathrm{i}\left(n+\frac{1}{2}\right) \omega t} \hat{O}^{n}\left(\left(1-\frac{2 k}{3 \omega^{2}} X\right)^{\frac{1}{4}} e^{\frac{2 \omega}{k}\left(3 \omega^{2} \sqrt{1-\frac{2 k}{3 \omega^{2}} X}+k X\right)}\right) .
$$

Consequently, applying the eigenvalue operator $\Sigma_{1}$ yields the positive part of the spectrum, i.e.:

$$
\Sigma_{1} \Phi_{n}=\omega\left(n+\frac{1}{2}\right) \Phi_{n}
$$

The negative part of the spectrum can be determined in the same way. We determine the invariant surface of $\Sigma_{3-}$, i.e.:

$$
G(t, X, \Phi)=g\left(t, \Phi \frac{e^{\frac{2 \omega}{k}\left(3 \omega^{2} \mathrm{i} \sqrt{\frac{2 k}{3 \omega^{2}} X-1}+k X\right)}}{\left(\frac{2 k}{3 \omega^{2}} X-1\right)^{\frac{1}{4}}}\right)
$$

that solved with respect to $\Phi$ yields:

$$
\Phi_{0^{-}}(t, X)=\tilde{T}(t)\left(\frac{2 k}{3 \omega^{2}} X-1\right)^{\frac{1}{4}} e^{-\frac{2 \omega}{k}\left(3 \omega^{2} \mathrm{i} \sqrt{\frac{2 k}{3 \omega^{2}} X-1}+k X\right)} .
$$

Substituting $\Phi_{0^{-}}(t, X)$ into (46) yields $\tilde{T}(t)=e^{\frac{\omega}{2} i t}$, i.e. the solution:

$$
\Phi_{0^{-}}(t, X)=\left(\frac{2 k}{3 \omega^{2}} X-1\right)^{\frac{1}{4}} e^{\frac{\omega}{2} \mathrm{i} t-\frac{2 \omega}{k}\left(3 \omega^{2} \mathrm{i} \sqrt{\frac{2 k}{3 \omega^{2}} X-1}+k X\right)} .
$$


The function $\Phi_{0^{-}}(t, X)$ (69) satisfies the boundary conditions (49).

$\Phi_{0^{-}}(t, X)$ is equivalent to the ground state since

$$
\left[\Sigma_{3-}, \Sigma_{\Phi_{0^{-}}}\right]=0
$$

and thus there are no states above $\Phi_{0^{-}}$.

The operator $\Sigma_{1}$ acts like an eigenvalue operator since:

$$
\Sigma_{1} \Phi_{0^{-}}=-\frac{\omega}{2} \Phi_{0^{-}}
$$

which yields the ground energy $E_{0^{-}}=-\omega / 2$, and consequently $\Phi_{0^{-}}(t, X)$ has a negative eigenvalue. i.e.:

Since $\left[\Sigma_{3+}, \Sigma_{\Phi_{0^{-}}}\right]=\Sigma_{\Phi_{1^{-}}}$, we explicitly determine the first negative excited state $\Phi_{1^{-}}(t, X)$,

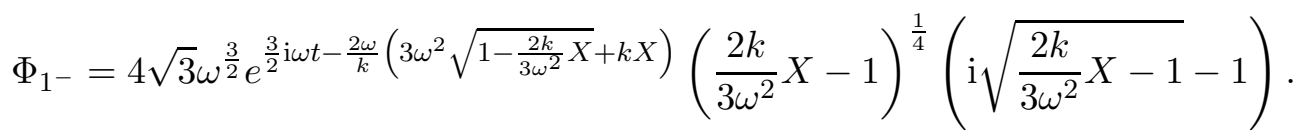

Applying $\Sigma_{1}$ to $\Phi_{1^{-}}(t, X)$ we get the corresponding eigenvalue:

$$
\Sigma_{1} \Phi_{1^{-}}=-\frac{3 \omega}{2} \Phi_{1^{-}}
$$

and by applying the commutator with $\Sigma_{3-}$ we indeed obtain:

$$
\left[\Sigma_{3-}, \Sigma_{\Phi_{1^{-}}}\right]=4 \omega k \Sigma_{\Phi_{0^{-}}} .
$$

Finally in analogy with the case of the positive part of the spectrum, we have the following recursive formula yielding all the eigenvalues and eigenfunctions:

$$
\begin{aligned}
{\left[\Sigma_{3+}, \Sigma_{\Phi_{n-1^{-}}}\right] } & =\Phi_{n^{-}} \partial_{\Phi}=\Sigma_{\Phi_{n^{-}}} \\
{\left[\Sigma_{3-}, \Sigma_{\Phi_{n^{-}}}\right] } & =\Phi_{n-1^{-}} \partial_{\Phi}=\Sigma_{\Phi_{n^{-}}} \\
\Sigma_{1} \Phi_{n^{-}} & =-\omega\left(n+\frac{1}{2}\right) \Phi_{n^{-}}
\end{aligned}
$$

\section{Comparison between the two quantization methods for (34)}

We compare the Noether symmetry quantization method applied to equation (34), as shown in the previous Section, with that used in [16]. Since the Hamiltonian (15) is a nonstandard one, the classical quantization rule (1) cannot be used. Therefore in [16] a simple modification of the quantization scheme proposed by von Roos [33, 34] for position-dependent masses was applied. Since the Hamiltonian (15) assumes the form

$$
H=\frac{x^{2}}{2 m(p)}+U(p)
$$

the authors of [16] introduced the following momentum-dependent mass:

$$
m(p)=\frac{1}{\omega^{2}\left(1-\frac{2 k}{3 \omega^{2}} p\right)}
$$


and also the following momentum-dependent potential:

$$
U(p)=\frac{9 \omega^{4}}{2 k^{2}}\left(\sqrt{1-\frac{2 k}{3 \omega^{2}} p}-1\right)^{2}
$$

Then the following Schrödinger equation was obtainedt:

$$
\begin{aligned}
2 \mathrm{i} \Psi_{t}+\omega^{2} & \left(1-\frac{2 k}{3 \omega^{2}} p\right) \Psi_{p p}-\frac{2 k}{3} \Psi_{p} \\
+ & {\left[\frac{4 k^{2} \alpha(\alpha+\beta+1)}{9 \omega^{2}\left(1-\frac{2 k}{3 \omega^{2}} p\right)}-\frac{9 \omega^{4}}{k^{2}}\left(\sqrt{1-\frac{2 k}{3 \omega^{2}} p-1}\right)^{2}\right] \Psi=0 . }
\end{aligned}
$$

where the constants $\alpha$ and $\beta$ are related with the other constant $\gamma$ by means of the condition

$$
\alpha+\beta+\gamma=-1
$$

as prescribed by the von Roos' method. Moreover the following further restriction was imposed:

$$
4 \alpha(\alpha+\beta+1)=-\frac{1}{4}
$$

in order to find the solution of equation (81) by means of Hermite differential equation since the spectrum is that described in the previous Section.

Unfortunately the eigenfunctions are singular on the right boundary $p=3 \omega^{2} / 2 k$ and consequently another Schrödinger equation was proposed by considering the following modified Hamiltonian :

$$
\bar{H}=m^{d} H m^{-d}
$$

and applying to it the von Roos' procedure. Thus it was found that $d$ must be equal to $-1 / 2$, and the following Schrödinger equation was obtained:

$$
\begin{aligned}
2 \mathrm{i} \Theta_{t}+\omega^{2} & \left(1-\frac{2 k}{3 \omega^{2}} p\right) \Theta_{p p} \\
+ & {\left[\frac{k^{2}}{12 \omega^{2}\left(1-\frac{2 k}{3 \omega^{2}} p\right)}-\frac{9 \omega^{4}}{k^{2}}\left(\sqrt{1-\frac{2 k}{3 \omega^{2}} p}-1\right)^{2}\right] \Theta=0 }
\end{aligned}
$$

which has bounded eigenfunctions and the same spectrum of equation (81).

We notice that equations (81) and (85) are actually related by the point transformation:

$$
\Psi(p, t)=\frac{\Theta(p, t)}{\sqrt{3 \omega^{2}\left(1-\frac{2 k}{3 \omega^{2}} p\right)}},
$$

\footnotetext{
${ }^{4}$ In [16] time-independent Schrödinger equations were derived.
} 
that eliminates the first-derivative in equation (81). Therefore the two equations admit the same finite Lie point symmetry algebra.

Moreover, the condition (83) on the constants $\alpha$ and $\beta$ is actually equivalent to require that the finite Lie point symmetry algebra of equation (81) - and consequently equation (85) - be six dimensional. Indeed the full Lie symmetry algebra is generated by the following operators:

$$
\begin{aligned}
& \Gamma_{1}=\left.\Xi_{1}\right|_{X=p}, \\
& \Gamma_{2}=\left.\Xi_{2}\right|_{X=p}+\left[\frac{\omega}{2} \sin (2 \omega t) \Psi \frac{2 \sqrt{1-\frac{2 k}{3 \omega^{2}} p}-1}{\sqrt{1-\frac{2 k}{3 \omega^{2}} p}}\right. \\
& \left.-\frac{9 \mathrm{i} \omega^{4}}{k^{2}} \cos (2 \omega t) \Psi\left(\sqrt{1-\frac{2 k}{3 \omega^{2}} p}-1\right)^{2}\right] \partial_{\Psi}, \\
& \Gamma_{3}=\left.\Xi_{3}\right|_{X=p}-\left[\frac{\omega}{2} \cos (2 \omega t) \Psi \frac{2 \sqrt{1-\frac{2 k}{3 \omega^{2}} p}-1}{\sqrt{1-\frac{2 k}{3 \omega^{2}} p}}\right. \\
& \left.+\frac{9 \mathrm{i} \omega^{4}}{k^{2}} \sin (2 \omega t) \Psi\left(\sqrt{1-\frac{2 k}{3 \omega^{2}} p}-1\right)^{2}\right] \partial_{\Psi} \\
& \Gamma_{4}=\left.\Xi_{4}\right|_{X=p}+\left[\frac{k}{6 \omega^{2}} \cos (\omega t) \frac{\Psi}{\sqrt{1-\frac{2 k}{3 \omega^{2}} p}}\right. \\
& \left.-\frac{3 \mathrm{i} \omega}{k} \sin (\omega t) \Psi\left(1-\sqrt{1-\frac{2 k}{3 \omega^{2}} p}\right)\right] \partial_{\Psi}, \\
& \Gamma_{5}=\left.\Xi_{5}\right|_{X=p}+\left[\frac{k}{6 \omega^{2}} \sin (\omega t) \frac{\Psi}{\sqrt{1-\frac{2 k}{3 \omega^{2}} p}}\right. \\
& \left.+\frac{3 \mathrm{i} \omega}{k} \cos (\omega t) \Psi\left(1-\sqrt{1-\frac{2 k}{3 \omega^{2}} p}\right)\right] \partial_{\Psi}, \\
& \Gamma_{6}=\Psi \partial_{\Psi}, \\
& \Gamma_{\psi}=\Psi(p, t) \partial_{\Psi}
\end{aligned}
$$

where $\Psi(p, t)$ satisfies equation (81)), and $\left.\Xi_{j}\right|_{X=p}(j=1,5)$ are the generators of the Noether symmetries (35) of equation (34) with $X$ replaced by $p$.

If $\alpha$ and $\beta$ do not satisfy condition (83) then equation (81) admits only $\Gamma_{1}, \Gamma_{6}$ and $\Gamma_{\psi}$ as Lie symmetries.

\section{$5 \quad$ Final remarks}

We have recalled an algorithm for quantization that requires the preservation of Noether symmetries in the passage from classical to quantum mechanics [12,13]. We have emphasized 
that it can be applied if the classical system of $N$ second-order equations admits the maximum number of Lie symmetries, namely $N^{2}+4 N+3$, and the linearizing transformation is time-independent.

Thus the derived Schrödinger equation is such that the independent-variables part of its admitted Lie symmetries corresponds to the Noether symmetries of the classical equations.

We have applied this quantization method to the linearizable Liénard equation (5) and compared our results with that found in [16].

We have shown that even in quantum mechanics whenever differential equations are involved, Lie and Noether symmetries have a fundamental role.

Indeed Noether symmetries give raise to the correct Schrödinger equation and its Lie symmetries can be algorithmically used to find the eigenvalues and eigenfunctions.

\section{Acknowledgement}

MCN acknowledges the support of the Italian Ministry of University and Scientific Research through PRIN 2010-2011, Prot. 2010JJ4KPA_004.

\section{References}

[1] D. Bjorken and S. D. Drell. Relativistic Quantum Mechanics. McGraw-Hill Book Co., New York, 1964.

[2] W. H. Louisell. Quantum Statistical Properties of Radiation. John Wiley \& Sons, New York, 1990.

[3] H. Weyl. Quantenmechanik und gruppentheorie. Zeitschrift für Physik, 46:1-46, 1927.

[4] L. van Hove. Sur certaines représentations unitaires d'un groupe infini de transformations. Memoires de la Académie Royale Belgique, Classe de Sciences, 26:1-102, 1951.

[5] M. Moshinsky and T.H. Seligman. Canonical transformations to action and angle variables and their representations in quantum mechanics. Ann. Phys., 114:243-272, 1978.

[6] A. Anderson. Canonical transformations in quantum mechanics. Ann. Phys., 232:292331, 1994.

[7] A. Brodlie. Nonlinear canonical transformations in classical and quantum mechanics. $J$. Math. Phys., 45:3413-3431, 2004.

[8] M. Błaszak and Z. Domański. Canonical transformations in quantum mechanics. Ann. Phys., 331:70-96, 2013.

[9] M. C. Nucci, P. G. L. Leach, and K. Andriopoulos. Lie symmetries, quantisation and c-isochronous nonlinear oscillators. J. Math. Anal. Appl., 319:357-368, 2006.

[10] H. Goldstein. Classical Mechanics, 2nd ed, Addison-Wesley, Reading, 1980.

[11] M. C. Nucci, P. G. L. Leach. Lie groups and quantum mechanics. J. Math. Anal. Appl., 406:219-228, 2013. 
[12] M. C. Nucci. Quantization of classical mechanics: shall we Lie? Theor. Math. Phys., 168:997-1004, 2011.

[13] M. C. Nucci. Quantizing preserving Noether symmetries, 2013 submitted.

[14] F. González-Gascón and A. González-López. Symmetries of differential equations. J. Math. Phys., 23:2006-2021, 1983.

[15] A. González-López. Symmetries of linear systems of second-order ordinary differential equations. J. Math. Phys., 29:1097-1105, 1988.

[16] V. Chithiika Ruby, M. Senthilvelan, and M. Lakshmanan. Exact quantization of a PT-symmetric (reversible) Liènard-type nonlinear oscillator. J. Phys. A: Math. Gen., 45:382002, 2012.

[17] B. Van Der Pol. Forced oscillations in a circuit with nonlinear resistance (receptance with reactive triode). Edinbourgh Dublin Phil. Mag., 3, 1927.

[18] A. Liénard. Études des oscillations entretenues. Rev. Gén. Electr., 23, 1928.

[19] Helmar Nunes Moreira. Liénard-type equations and the epidemiology of malaria. Ecological Modelling, 60:139-150, 1992.

[20] V. K. Chandrasekar, M. Senthilvelan, and M. Lakshmanan. Unusual Liénard-type nonlinear oscillator. Phys. Rev. E, 72:066203, 2005.

[21] V. K. Chandrasekar, M. Senthilvelan, A. Kundu, and M. Lakshmanan. A nonlocal connection between certain linear and nonlinear ordinary differential equations/oscillators. $J$. Phys. A: Math. Gen., 39:9743, 2006.

[22] V. K. Chandrasekar, J. H. Sheeba, R. Gladwin Pradeep, R. S. Divyasree, and M. Lakshmanan. A class of solvable coupled nonlinear oscillators with amplitude independent frequencies. Phys. Lett. A, 376:2188-2194, 2012.

[23] R. Iacono and F. Russo. Class of solvable nonlinear oscillators with isochronous orbits. Phys. Rev. E, 83:027601, 2011.

[24] F. M. Mahomed and P. G. L. Leach. The linear symmetries of a nonlinear differential equation. Quaest. Math., 8:241-274, 1985.

[25] P. G. L. Leach. First integrals for the modified Emden equation $\ddot{q}+\alpha(t) \dot{q}+q^{n}=0 . \quad J$. Math. Phys., 26:2510-2514, 1985.

[26] P. G. L. Leach, M. R. Feix, and S. Bouquet. Analysis and solution of a nonlinear secondorder differential equation through rescaling and through a dynamical point of view. $J$. Math. Phys., 29:2563-2569, 1988.

[27] S. Lie. Vorlesungen über Differentialgleichungen mit bekannten infinitesimalen Transformationen. Teubner, Leipzig, 1912.

[28] M.C. Nucci and K.M. Tamizhmani. Lagrangians for dissipative nonlinear oscillators: the method of Jacobi Last Multiplier. J. Nonlinear Math. Phys., 17:167-178, 2010. 
[29] C. G. J. Jacobi. Sul principio dell'ultimo moltiplicatore, e suo uso come nuovo principio generale di meccanica. Giornale Arcadico di Scienze, Lettere ed Arti, 99:129-146, 1844.

[30] C. G. J. Jacobi. Theoria novi multiplicatoris systemati æquationum differentialium vulgarium applicandi. J. Reine Angew. Math., 27:199-268, 1844.

[31] C. G. J. Jacobi. Theoria novi multiplicatoris systemati æquationum differentialium vulgarium applicandi. J. Reine Angew. Math., 29:213-279 and 333-376, 1845.

[32] C. G. J. Jacobi. Vorlesungen über Dynamik. Nebst fünf hinterlassenen Abhandlungen desselben herausgegeben von A. Clebsch. Druck und Verlag von Georg Reimer, Berlin, 1884 .

[33] O. von Roos. Position-dependent effective masses in semiconductor theory. Phys. Rev. B, 27:7547-7552, Jun 1983.

[34] O. von Roos and H. Mavromatis. Position-dependent effective masses in semiconductor theory. II. Phys. Rev. B, 31:2294-2298, Feb 1985.

[35] M.C. Nucci. Interactive reduce programs for calculating Lie point, non-classical, LieBäcklund, and approximate symmetries of differential equations: manual and floppy disk. In N.H. Ibragimov, editor, CRC Handbook of Lie Group Analysis of Differential Equations, volume III: New Trends, chapter 14. CRC Press, Boca Raton, 1996.

[36] P.M. Mathews and M. Lakshmanan. On a unique nonlinear oscillator. Quart. Appl. Math., 32:215-218, 1974.

[37] M. Lakshmanan and S. Rajasekar. Nonlinear Dynamics: Integrability, Chaos and Patterns. Advanced Texts in Physics. Springer-Verlag, Berlin, 2003.

[38] P. G. L. Leach. The solution of some quantum nonlinear oscillators with the common symmetry group $S L(2, R)$, J. Phys. A: Math. Gen., 38:1543-1552, 2005.

[39] K. Andriopoulos and P. G. L. Leach. Wave-functions for the time-dependent linear oscillator and Lie point symmetries, J. Phys. A: Math. Gen., 38:4365-4374, 2005.

[40] M. C. Nucci and P. G. L. Leach. Gauge variant symmetries for the Schrödinger equation, Il Nuovo Cimento B, 123:93-101, 2008.

[41] M. C. Nucci and P. G. L. Leach. An algebraic approach to laying a ghost to rest. Phys. Scripta, 81:055003, 2010. 